\title{
Pobreza e HIV/AIDS: aspectos antropológicos e sociológicos
}

\author{
Poverty and HIV/AIDS: \\ anthropological and sociological aspects
}

Richard Parker 1,2,3

Kenneth Rochel de Camargo J r. 1,3

\footnotetext{
1 Instituto de Medicina Social, Universidade do Estado do Rio de Janeiro. Rua São Francisco Xavier 524, 7o andar, Bloco D, Rio de Janeiro, RJ 20559-900, Brasil. 2 School of Public Health, Columbia University, 600 West 168th Sreet, 7th floor, New York, NY, 10032, EUA

3 Associação Brasileira Interdisciplinar de AIDS. Rua da Candelária 79, 10 o andar, Rio de Janeiro, R 20091-020, Brasil.
}

\begin{abstract}
Focusing on the HIV/AIDS epidemic as a summation of several epidemics coexisting in the same space and drawing on Brazilian epidemiological data, we argue that the epidemic there shows variations al ready described el sewhere, such as feminization, pauperization, juvenization and interiorization, as a result of the deep inequalities characteristic of Brazilian society. We then examine the contributions of three bodies of sociol ogi cal and anthropological literature related to HIV/AIDS: 1) soci ological research and theory on the impact of recent gl obal economic restructuring and social transformation, and its relationship to public health issues; 2 ) the cross-cultural and cross-national anthropological and sociological literature on structural factors shaping the course of the epidemic in different settings; and 3) the body of anthropological and soci ol ogi cal research on the synergistic effects of HIVIAIDS, social exclusion, and related social problems in pockets of extreme poverty in the large cities of core countries. We conclude that prevention policies for HIV/AIDS should deal comprehensi vely with di verse dimensions that determine differential vulnerabilities to the epidemic, thus requiring substantial social transformations.

Key words Acquired Immunodeficiency Syndrome; HIV; Poverty; Medical Anthropology; Social Conditions
\end{abstract}

Resumo A partir da perspectiva das várias epi demias de HIV/AIDS que coexistem em mesmo espaço, bem como dos dados epidemi ológi cos do Brasil, acredita-se serem observáveis as variações já descritas alhures - feminilização, pauperização, juvenilização e interi orização - como resultado das profundas desigual dades da soci edade brasil eira. Foram examinadas as contri bui ções de três vertentes de anál ise dos aspectos sócio-econômi cos da AIDS: 1) pesqui sas e teori as soci ológi cas a respei to do impacto da reestruturação econômica e transformação social gl obal recentes e sua relação com a saúde pública; 2) li teratura transcultural e transnacional em antropologi a e soci ol ogia dedicada aos fatores estruturais que conformam o curso da epidemia em diferentes conjunturas; e 3) corpo de pesquisas antropológi cas e soci ológi cas concernentes aos efeitos sinérgi cos do HIV/AIDS, exclusão social, e probl emas sociais associados nos bolsões de extrema pobreza encontrados nas grandes cidades de países centrais. Concl ui-se que as políticas de prevenção do HIV/AIDS devem abordar, de forma integrada, as várias dimensões que determinam as diferenciadas vul nerabilidades à epi demia, dependendo, portanto, de transformações sociais substantivas.

Palavras-chave Síndrome da Imunodeficiência Adquirida; Pobreza; HIV; Antropologia Médica; Condições Sociais 
Introdução

Há quase dez anos, Jonathan Mann et al. (1993, 1996) já chamavam a atenção para o fato de que se designava correntemente como "a" epidemia de HIV/AIDS aquilo que, na verdade, era o resultado observável, em nível macro, de dezenas, talvez centenas, de epidemias ocorrendo em paralelo, acometendo segmentos diversos da sociedade com padrões de disseminação e velocidades variadas, dependentes de uma série de fatores.

Embora aqueles autores estivessem analisando os eventos em escala global, poderíamos aplicar, mutatis mutandis, o mesmo raciocínio para o Brasil, país de dimensões continentais com importantes disparidades sociais, econômicas e demográficas em sua população. É de esperar, portanto, que tais disparidades - e os vários Brasis que elas delimitam - reflitam-se também na forma como o HIV se propaga em nossa população. Dito de outra forma, estas diferentes características conformariam populações diferentes, ainda que em mesmo território, com variações quanto à probabilidade de que seus componentes viessem a infectar-se pelo HIV, configurando, portanto, diferentes vulnerabilidades à infecção pelo HIV e, por conseguinte, à AIDS.

Pode-se supor que estas variações se reflitam nos dados coletados em nosso meio a respeito do HIV/AIDS, como examinaremos a seguir. Deve-se ter em mente, de qualquer modo, que as mesmas desigual dades que explicam diferentes vulnerabilidades ao HIV/ AIDS também têm efeitos sobre a possibilidade de indivíduos acometidos serem captados pela rede de saúde e, em conseqüência disso, serem oficialmente notificados como "casos". Semelhante problema ocorre, por exemplo, no registro de óbitos, em que algumas regiões do país apresentam número el evado de óbitos identificados como "causa desconhecida", o que é usualmente interpretado como deficiência do sistema de saúde local. Sendo assim, é preciso que os dados disponíveis sejam sempre observados com al guma cautela, pois não só devemos esperar erros, como também presumir que estes erros, longe de serem aleatórios, sejam enviesados de modo diferenciado por fatores sociais e econômicos.

Há outros problemas para a análise destas informações. Os dados aqui estudados referem-se - a não ser quando explicitamente dito de outra forma - ao Boletim Epidemi ol ógi co AIDS (CNDST/AIDS, 1999).

As fichas de notificação não trazem muitas informações acerca de variáveis sócio-econô- micas - com exceção do grau de instrução - e, nas informações consolidadas, este indicador deixa de ser informado para os menores de 13 anos, pois o grau de instrução da mãe é considerado como boa aproximação daquela variável. Outras informações que poderiam permitir análise mais criteriosa - como, por exemplo, a cor ou etnia dos acometidos - também não são captadas. Do ponto de vista da distribuição espacial, a disponibilização da informação agregada ao nível de cidade impõe, da mesma forma, certas limitações; pense-se, por exemplo, na infinidade de situações apresentadas por cidades como Rio de Janeiro e São Paulo.

Impõe-se, portanto, uma primeira consideração: há necessidade de revisão do instrumento de notificação e/ou a realização de estudos epidemiológicos adicionais que visem especificamente à determinação do(s) perfil(is) sócio-econômico(s) da população afetada pelo HIV/ AIDS. Não obstante, as informações disponíveis permitem algumas observações, ainda que não a formulação de modelos matemáticos precisos (ver, p. ex., Gomes, 1998).

Uma primeira observação que pode ser feita, no que concerne à evolução da epidemia no Brasil desde a identificação dos primeiros casos, refere-se à distribuição geográfica dos mesmos.

Embora a incidência em termos absolutos - em especial, a incidência acumulada - ainda mostre importante concentração nas duas maiores cidades brasileiras - isto é, São Paulo e Rio de Janeiro - a incidência proporcional (com relação ao número de habitantes) traz algumas surpresas (Tabela 1) (CNDST AIDS, 1999). Das dez cidades com maiores incidências no país, apenas uma delas é capital de Estado (Florianópolis, em 4o lugar); São Paulo está em 180 lu= gar nesta lista e o Rio de Janeiro, em 25o.

Assim, ainda que concordando com CastiIho \& Chequer (1997) no sentido de que não é possível apontar intensa interiorização e, menos ainda, "ruralização" da epidemia (dadas as características da população brasileira - tornada maciçamente urbana ao longo dos últimos 40 anos - e as formas de transmissão do HIV, seria surpreendente observar-se "ruralização" da epidemia), com certeza é viável assinalar importante disseminação geográfica, tendência que provavel mente deverá acentuar-se no futuro (ver Bastos, 1996). Tal propagação não se faz mecanicamente, e é possível explorar mecanismos que expliquem esta difusão diferencial. Um dos processos com provável relevância é, por exemplo, a distribuição e consumo de drogas de uso recreacional (não-terapêutico), o que teria importância diferenciada 
em cidades como Santos e Itajaí (Bastos, 1996; Castilho \& Chequer, 1997).

Outra característica importante da progressão da epidemia diz respeito à distribuição por sexo. Como já foi notado por diversos autores, a proporção entre indivíduos acometidos do sexo masculino e feminino - que já foi de 17 para 1, em 1983 - esteve em 2 para 1 nos dois últimos anos. A ressalva feita por Castilho \& Chequer (1997:25), de que parte significativa dos casos em mulheres seria computada na categoria "usuário de drogas injetáveis" (UDI) não mais se aplica; em 1998/ 1999, de 6.055 casos notificados em mulheres, 3.855 (63,7\%) foram classificados na categoria de transmissão sexual e apenas 533 (8,8\%), como UDI.

Mais ainda, a segunda principal causa de óbito em mulheres entre 20 e 49 anos de idade no Brasil eram, já em 1995, os transtornos que envolviam os mecanismos imunitários (Gomes, 1998), e esta categoria atualmente é a primeira nesta faixa etária e sexo em São Paulo (Gomes, 1998). Sendo assim, a chamada "feminização" da epidemia não é mais mera conjetura.

Um último aspecto de interesse a ser abordado nesta introdução diz respeito à distribuição segundo grau de instrução, que, como dito anteriormente, constitui o único indicador dentre aqueles disponibilizados pelo sistema de notificação com alguma correlação com variáveis sócio-econômicas, ainda que com restrições: os dados consolidados divulgados pelo Boletim Epi demiológi co apresentam este indicador apenas para indivíduos com idade maior ou igual a 19 anos (CNDST/ AIDS, 1999). Comparando-se apenas dois períodos citados no Bol etim, poderíamos compor a Tabela 2.

Com todas as ressalvas que devem ser feitas quanto ao ainda elevado número de casos sem informação a respeito do grau de instrução, bem como acerca da magnitude diversa das incidências nos dois períodos, parece-nos razoável considerar a existência de tendência ao acometimento de indivíduos com menor grau de instrução, o que, por sua vez, seria indicativo da propagação da epidemia em direção aos segmentos mais desfavorecidos da sociedade, já descrita por muitos como pauperização.

Um entendimento mais completo das tendências epidemiológicas que têm sido identificadas na evolução da epidemia de HIV/AIDS no Brasil, depende de nossa capacidade de contextualizá-las em relação a padrões sociológicos e antropológicos mais amplos e, com base nisso, definir agenda para pesquisa e intervenção que seja capaz de responder à pauperização, à feminização e à interiorização da epidemia de AIDS no Brasil, sem descuidar,
Tabela 1

Incidência de casos de AIDS por $100 \mathrm{mi}$ habitantes, cidades com os dez maiores coeficientes de incidência. Brasil, 1999.

\begin{tabular}{lc}
\hline Cidade & $\begin{array}{c}\text { Coeficiente } \\
\text { de Incidência }\end{array}$ \\
\hline Itajaí (SC) & 854,8 \\
Balneário Camboriú (SC) & 721,4 \\
São J osé do Rio Preto (SP) & 665,3 \\
Florianópolis (SC) & 651,9 \\
Santos (SP) & 636,3 \\
Ribeirão Preto (SP) & 626,6 \\
Bebedouro (SP) & 579,8 \\
Barretos (SP) & 526,8 \\
Catanduva (SP) & 474,7 \\
Brasil & 218,0
\end{tabular}

Fonte: Boletim Epidemiológico - CNDST/AIDS, 1999.

Tabela 2

Distribuição percentual dos casos de AIDS

em dois períodos selecionados. Brasil, 1999.

\begin{tabular}{|c|c|c|}
\hline Grau de instrução & $\% 1985$ & $\% 1998 / 9$ \\
\hline analfabeto & 0,6 & 4,8 \\
\hline 10 grau & 13,3 & 55,6 \\
\hline 20 grau & 10,2 & 14,8 \\
\hline superior & 20,1 & 6,6 \\
\hline ignorado & 57,4 & 18,2 \\
\hline
\end{tabular}

Fonte: Boletim Epidemiológico - CNDST/AIDS, 1999.

neste processo, de grupos que, embora minoritários na sociedade brasileira, ainda permanecem particularmente vulneráveis à mesma.

Pelo menos três áreas na literatura sociológica e antropológica parecem ser especialmente importantes neste aspecto:

1) pesquisas e teorias sociológicas recentes voltadas ao impacto da reestruturação econômica e transformação social global ao final do século vinte, e a relação deste processo de mudança com questões de saúde pública como a HIV/AIDS;

2) literatura transcultural e transnacional em antropologia e sociologia relativas aos fatores estruturais que conformam o curso da epidemia de HIV/ AIDS em diferentes conjunturas; e 
3) corpo de pesquisas antropológicas e sociológicas que tratam dos efeitos sinérgicos do HIV/AIDS, pobreza intensa, e problemas sociais associados nos bolsões de extrema pobreza encontrados nas grandes cidades de países como os Estados Unidos.

Nas próximas páginas, revisamos rapidamente cada uma dessas áreas e examinamos as implicações que podem ter para entendimento mais completo das tendências que têm sido identificadas na epidemia de HIV/AIDS no Brasil. Na conclusão sugerimos as maneiras pelas quais esta literatura já existente pode ajudar a conformar agenda de pesquisa e ação dirigida à HIV/AIDS no Brasil.

Contexto histórico: globalização

e emergência do "Quarto Mundo"

Talvez o fator relativo ao contexto mais importante a ser tomado em conta ao buscar-se o entendimento da evolução global da epidemia de HIV/AIDS seja a transformação amplamente simultânea, também em nível global, da economia internacional - bem como uma série de transformações subseqüentes nas estruturas de sociedades, comunidades e famílias. Como tem sido documentado internacionalmente, as décadas finais do século $X X$ caracterizam-se por complexos processos de globalização e de reestruturação econômica que começam a acelerar-se, grosso modo, no início da década de 70 (por volta de 1973).

Embora estes processos sejam complexos e diversos, e a transição esteja longe de ter sido completada, a tendência geral tem sido em direção a uma passagem fundamental do que é costumeiramente descrito como o regime Keynesiano-Fordista do capitalismo industrial para o que Manuel Castells, entre outros, descreveu como "capitalismo informacional" como modo dominante de desenvolvimento ao redor do mundo neste fim de século (Castells, 1999).

Mesmo sem entrar em detal hes a respeito de todos os aspectos desta nova forma de organização econômica capitalista, vale a pena enfatizar al guns de seus elementos. Em particular, observa-se uma substituição dos tradicionais materiais brutos da produção industrial pelo controle sobre o processamento da informação como a característica-chave da produção e acumulação capitalistas globais (Castells, 1999). Esta substituição conectou-se, por sua vez, a nova ênfase na flexibilidade (acumulação flexível, esquemas flexíveis de produção, trabalho e vínculos empregatícios flexíveis e assim por diante) bem como a formas de orga- nização social e econômica em rede (Castells, 1999; Sennett, 1999). A nova forma de organização tem, como característica, a rápida circulação de capital ao redor do globo através do sistema financeiro internacional, e o fato de ser hoje possível todo o mercado global (e para o sistema global de produção) funcionar como entidade única, trabalhando e interagindo em tempo real (a característica que mais claramente distingue a fase da assim denominada "globalização" de qualquer etapa prévia do "sistema mundial", como definida por Wallerstein e outros) (Harvey, 1989; Watters, 1995; Castells, 1999).

A partir de uma perspectiva sociológica e antropológica, talvez a conseqüência central deste conjunto de transformações econômicas tenha sido a extensão com que, aparentemente, as elites ao redor do mundo se conectaram, ao mesmo tempo em que foram criadas novas formas de exclusão social e extremos de desigualdade, diferenciais de renda, pobreza e miséria em todas as sociedades, de Norte a Sul.

Com efeito, parece que estamos vivendo, ao término deste século, nova etapa histórica, na qual divisões prévias entre Norte e Sul, Primeiro e Terceiro Mundos, e assim por diante, estão sendo demolidas, mas na qual, simultaneamente, está se dando a emergência do que Castells chama de "Quarto Mundo": segmentos populacionais importantes na maior parte das sociedades - talvez, mesmo, continentes inteiros, no caso da África Sub-Sahariana - que são essencialmente irrelevantes para os interesses básicos - tanto em termos de produção quanto de consumo - do sistema do capital informacional (Castells, 1999).

Esta fase recente da globalização tem-se caracterizado, portanto, por profunda acentuação dos processos de diferenciação social, por um lado, e por relações de distri bui ção e consumo, por outro. A organização social da diferenciação ou desigualdade na distribuição tem sido tipificada por crescente polarização entre ricos e pobres, na qual os ricos tornam-se cada vez mais ricos e os pobres cada vez mais pobres, com setores intermediários - a classe média - gradativamente desaparecendo. Esta poIarização, por sua vez, tem sido associada ao crescimento significativo da miséria pura e simples (extrema pobreza, usualmente definida como menos que $50 \%$ da renda que definiria o nível de pobreza em dada sociedade), em todos os países em qualquer parte do mundo. Ao mesmo tempo, a diferenciação social nas relações de produção tem-se caracterizado pela crescente individual ização do trabalho (tipificada pela decadência, em força e significação, 
dos sindicatos e pelo rápido crescimento do setor informal da economia, como os camelôs e biscateiros no caso do Brasil), a superexploração de trabalhadores (exemplificada pelo crescimento do trabalho infantil), a crescente exclusão de grupos populacionais significativos do mercado de trabalho (adolescentes do sexo masculino membros de minorias em países como o Brasil e os Estados Unidos), da mesma forma que a integração perversa destes mesmos setores no mundo paralelo da economia criminosa (Castells, 1998).

Estas tendências básicas foram identificadas em escala planetária tanto nos chamados países desenvolvidos quanto em desenvolvimento. Também foram ligadas ao que poderia ser descrito como progressiva feminização da pobreza e da mi séria. Embora as mulheres venham sendo incorporadas ao mercado de trabalho em proporções sem precedentes nas últimas décadas (ao menos, em parte, através de famílias de classe média, nas quais a presença de um segundo membro com fonte de renda tornou-se necessária como estratégia de manutenção do padrão de vida em contexto de crescente polarização econômica e salários reais declinantes), a face da pobreza e, em particular, da miséria, tem-se tornado, de modo crescente, a face das mulheres excluídas não apenas devido à opressão baseada em classe e etnia, mas também à opressão baseada em gênero.

Tal opressão tem sido reforçada, acima de tudo, pela rápida redução - que vem ocorrendo em todo o mundo - nos programas de saúde, educação e proteção social pela adoção de políticas sociais e econômicas neoliberais orientadas à produção de crescente integração no sistema capitalista global (simultaneamente, minimizando o papel do Estado). Tais transformações têm impactado desproporcionalmente as vidas das mulheres, acentuando a feminização da pobreza e da miséria mesmo em meio a uma série de ganhos importantes em termos do feminismo e da conquista de direitos civis e políticos adicionais na maioria dos países.

O fato de que a pandemia global de HIV/ AIDS emergiu precisamente durante o mesmo período histórico em que tais mudanças econômicas e sociais maciças tiveram (e têm tido) lugar, pode ter sido originalmente um acidente histórico, mas a relação entre a evolução da epidemia e as conseqüências disseminadas destas mudanças históricas não é, de modo algum, coincidência.

Por um lado, al guns dos suportes técnicos que permitiram a transformação radical do capitalismo, "encolhendo" o mundo, provavel- mente foram também facilitadores da propagação da epidemia. Por outro lado, os impactos negativos da globalização aqui apontados - em especial, aquilo que Castells denomina “integração perversa" (Castells, 1998) - isto é, a produção da marginalização e mesmo da criminalidade como parteintegrante da nova ordem capitalista mundial, e não como mero desvio ou acidente de percurso - são os ingredientes estruturais da produção de susceptibilidades crescentes à infecção - a vulnerabilidade - e da incapacidade crescente de indivíduos e sociedades cuidarem de seus doentes, de modo geral, e dos acometidos pelo HIV/AIDS, em particular.

Pobreza e (sub)desenvolvimento na literatura internacional

Ao longo da última década, pesquisadores têm documentado certo número de fatores estruturais que facilitam a transmissão do HIV e sua concentração em áreas geográficas e populações particulares (Ayres, 1994; Sweat \& Denison, 1995; Tawil et al., 1995; Turshen, 1995; Aggleton, 1996; Caraël et al., 1997; Singer, 1998). Estes fatores podem ser agrupados em três categorias distintas, mas interconectadas:

1) (sub)desenvolvimento econômico e pobreza;

2) mobilidade, incluindo migração, trabalho sazonal, e convulsão social em razão de guerras e de instabilidade política, que interagem freqüentemente com a pobreza, condicionando a vulnerabilidade relacionada ao HIV/ AIDS;

3) desigualdades de gênero, que também interagem com a pobreza (e a contínua feminização da pobreza), colocando as mulheres, bem como homens desviantes com relação ao gênero (por exemplo, travestis), em situações de vulnerabilidade acentuada à infecção pelo HIV.

As pesquisas disponíveis mostram que, apesar das características singulares de cada epidemia local de HIV/ AIDS, as mesmas estruturas e processos podem ser encontrados na África (Schoepf, 1988, 1992a, 1992b; Wilson et al., 1990; Bond \& Vincent, 1991; Jochelson et al., 1991; Anarfi, 1993; Bassett, 1993; Akeroyd, 1994; Decosas, 1996; Decosas et al., 1995; Webb, 1997; Romero-Daza \& Himmelgreen, 1998; Turshen, 1998), assim como na Ásia (Tan, 1993; Archavanitkul \& Guest, 1994; Kammerer et al., 1995; Symonds, 1998) e na América Latina e Caribe (Daniel \& Parker, 1991, 1993; Farmer, 1992, 1995; Farmer et al., 1993; Lurie et al., 1995; Ayres, 1996; Paiva, 1996, 1999; Kreniske, 1997; Susser $\&$ Krenishe, 1997), da mesma forma que em certos grupos e comunidades na América do Nor- 
te (Des Jarlais et al., 1992; Lindenbaum, 1998; Singer, 1998).

Um dos temas-chave examinados nesta literatura é a conexão entre o subdesenvolvimento econômico e a vulnerabilidade ao HIV/ AIDS. Com efeito, o próprio processo de desenvolvimento cria, com freqüência, formas de deslocamento social, as quais, por seu turno, produzem ações e práticas sociais que aumentam o risco de infecção pelo HIV. Exemplo excelente, mostrando as conseqüências não intencionais do desenvolvimento econômico em relação ao HIV/ AIDS, é a análise histórica empreendida por Joseph Decosas de como a construção da barragem Akosombo, em Gana, durante os anos 60, contribuiu para a epidemia de HIV/ AIDS no povo Krobo, de Gana, nos anos 80 e 90 (Decosas, 1996).

Durante a construção da barragem, muitos homens Krobo se deslocaram rio abaixo para trabalhar no projeto, enquanto diversas muIheres Krobo passaram a fornecer serviços, inclusive trocas econômico-sexuais, para os homens que trabal havam no canteiro de obras da barragem. Quando a criação do Lago Volta destruiu a base agrícola dos Krobo, um número considerável destas mulheres, e mais tarde, suas filhas, emigraram para trabalhar como prostitutas, e os ganhos com o trabalho sexual se tornaram importante fonte de desenvolvimento nesta região. Estas duas gerações de mulheres têm elevada incidência de HIV. Hoje, com a melhora das perspectivas econômicas de Gana, os ganhos de mulheres trabalhando em outros países se tornaram menos relevantes, uma quantidade menor de moças jovens está se tornando trabalhadora do sexo, e a incidência do HIV entre as mulheres Krobo jovens está se aproximando das baixas taxas observadas no resto de Gana.

A análise de Decosas demonstra as dificuldades em estabelecer o mecanismo de associação entre o desenvolvimento econômico e o HIV, uma vez que causas e efeitos, tal como custos e benefícios, são dinâmicos e se desenvolvem ao longo de décadas.

A pesquisa antropológica de longo prazo de Paul Farmer, no Haiti, documenta de modo similar como os deslocamentos causados por iniciativas de desenvolvimento de larga escala podem impulsionar a disseminação da infecção pelo HIV (Farmer, 1992). De modo mais amplo, políticas internacionais e intergovernamentais de desenvolvimento têm sido associadas à desintegração de estruturas sócio-econômicas tradicionais e à acentuação de desigualdades sócio-econômicas, o que, por sua vez, tem contribuído de forma significativa para a severidade da epidemia em todos os países em desenvolvimento. Com efeito, a própria pobreza tem sido identificada como, possivelmente, a força sócio-econômica central na determinação da epidemia, e, virtualmente, toda a literatura estrutural e ambiental tem enfatizado a poderosa interação entre a pobreza e outras formas de desigualdade, instabilidade e discriminação social na produção da disseminação do HIV (Farmer et al., 1996; Singer, 1998).

Um número significativo de pesquisas tem associado a pobreza à migração eà mobilidade, sugerindo que a migração, em resposta a imperativos econômicos, está associada a maiores incidências de vulnerabilidade ao HIV em uma variedade de contextos e lugares, aí incluindose: trabalhadores sazonais, no sul da África (Romero-Daza \& Himmelgreen, 1998) e na África Ocidental (Decosas et al., 1995); migrantes da República Dominicana, nos EUA (Kreniske, 1997); migrantes de áreas rurais para urbanas, no Haiti (Farmer, 1992, 1999) e Zaire (Schoepf, 1992a); filipinos, trabalhando por empreitada no exterior (Tan, 1993); trabalhadoras do sexo, na Tailândia (Archavanitkul \& Guest, 1994), em Gana (Anarfi, 1993), no Zimbabwe (Wilson et al., 1990) e nas Filipinas (Tan, 1993), assim como trabal hadores do sexo e outros homens que fazem sexo com homens no Brasil (Larvie, 1997; Parker, 1993, 1997).

Os nexos causais subjacentes à conexão entre mobilidade e disseminação do HIV são complexos. Trabalhadores migrantes, por exemplo, freqüentam trabalhadoras do sexo com regularidade (freqüentemente, elas próprias também migrantes) e/ ou estabelecem famílias secundárias no campo de trabalho, levando a aumento das infecções sexualmente transmissíveis (IST), inclusive da infecção pelo HIV, em locais usual mente carentes de serviços adequados de atenção à saúde. De volta à comunidade de origem, as mulheres defrontam-se com demandas econômicas e emocionais severas, que tentam equacionar através do trabalho na agricultura e, às vezes, mediante a prática do sexo comercial. Finalmente, uma vez que trabalhadores e trabalhadoras migrantes deslocam-se continuamente entre dois ou mais locais, o HIV pode-se disseminar de áreas com alta incidência a outras onde esta incidência é menor ou mesmo previamente inexistente.

Tais exemplos da relação entre pobreza, migração/mobilidade e vulnerabilidade ao HIV sugerem que os fatores político-econômicos que impulsionam a epidemia de HIV/AIDS estão também intimamente ligados à organização social de estruturas de gênero e sexualidade, cujas hierarquias fazem das mulheres - em 
especial, aquelas dos segmentos de baixa renda - extremamente vulneráveis à infecção pelo HIV. Não obstante, têm sido raros os estudos aprofundados a respeito do gênero e da sexualidade como fatores estruturais - em contraposição a estudos comportamentais - na determinação da transmissão do HIV.

As inadequações resultantes das pesquisas e intervenções em AIDS orientadas às mulheres levaram alguns pesquisadores a olhar mais atentamente para os sistemas de gênero e sexualidade, de modo a desenvolver opções de redução de risco para o HIV mais realistas e efetivas para as mulheres (Schoepf, 1992a, 1992b; Schoepf et al., 1991; Gupta \& Weiss, 1993; Elias \& Heise, 1994; Michal-Johnson, 1994; Heise \& Elias, 1995; Kammerer et al., 1995; Symonds, 1998; Zoysa et al., 1996).

Heise \& Elias (1995), por exemplo, argumentam que a estratégia em três frentes da maior parte dos programas de prevenção da AIDS (quais sejam: redução de parceiros, promoção do uso dos preservativos e tratamento das IST) é inadequada para proteger a maior parte das mulheres do mundo, as quais são pobres e não detêm o poder de negociação dos encontros sexuais. Mais ainda, a associação dos preservativos com a desconfiança e com as falhas de comunicação entre homens e mulheres no que diz respeito aos assuntos sexuais e reprodutivos, além da falta de percepção da vulnerabilidade ao HIV, limitam ainda mais a capacidade de muitas mulheres praticarem o sexo seguro (Michal-Johnson, 1994; Heise \& Elias, 1995; Parker \& Galvão, 1995; Zoysa et al., 1996; Barbosa, 1999), situação agravada pela falta de tecnologias de prevenção do HIV controladas peIas mulheres (Stein, 1990; Elias \& Heise, 1994; ABIA, 1999).

$\mathrm{Na}$ literatura de gênero e sexualidade existem várias análises etnográficas impressionantes que iluminam os fatores culturais e políticos subjacentes à vulnerabilidade ao HIV. Kammerer et al. (1995), por exemplo, descrevem como a penetração do Estado e do capitalismo produziram colapso da economia das tribos das montanhas na periferia do norte da Tailândia. Como resultado, jovens têm migrado para as cidades do vale para trabalhar - às vezes, na prostituição -, enquanto, ao mesmo tempo, a sexualidade montanhesa tradicional, com seus valores de "vergonha, nomear e culpar" (no inglês, "shame, nameand blame"), tem constituído obstáculo significativo à adoção de medidas preventivas contra o HIV.

Symonds, escrevendo igual mente a respeito do norte da Tailândia, explica de forma similar a vulnerabilidade ao HIV entre os Hmong como produto de combinação de fatores políticos, econômicos e culturais, incluindo-se a entrada dos $\mathrm{Hmong}$ - originários das terras altas - nos mercados das terras baixas, o crescimento da indústria do sexo, o uso de drogas injetáveis, o racismo e a discriminação contra os Hmong por parte da maioria Thai e o duplo padrão sexual (Symonds, 1998).

A análise feita por Schoepf de histórias de vida de mulheres no Zaire também evidencia que o HIV não é disseminado por práticas sexuais exóticas, mas por respostas normais a problemas do cotidiano, tais como dificuldades econômicas substanciais e incerteza (Schoepf, 1992b).

Todos os autores aqui citados promovem formas participativas e colaborativas de pesquisa-ação com mulheres vulneráveis como modo de redefinir os papéis sociais de gênero e as condições sócio-econômicas que contribuem para a disseminação do HIV (Schoepf et al., 1991; Schoepf, 1992a, 1992b; Kammerer et al., 1995; Symonds, 1998).

Final mente, ainda que um volume consideravelmente menor de pesquisas tenha sido realizado no que se refere aos homens que fazem sexo com homens em países em desenvolvimento, os achados mostram que a vulnerabilidade ao HIV relacionada à desigualdade de gêneros e ao sexismo também estão presentes quase universalmente em relações entre parceiros do mesmo sexo (McKenna, 1996).

Neste contexto, as estruturas de desigualdade de gênero são tipicamente replicadas através da estigmatização dos homens homossexuais particularmente efeminados e pessoas transexuais que, com freqüência, têm poucas opções fora do trabalho em sexo e são objeto de violências físicas socialmente sancionadas (Parker, 1993). Estes estudos sugerem que os homens que fazem sexo com homens estão presentes em todas as sociedades e que opressões múltiplas - incluindo-se a pobreza, o racismo, a desigualdade de gênero e a homofobia - interagem de forma sistemática, fazendo com que tais homens fiquem em situação de acentuada vulnerabilidade à infecção pelo HIV (Parker et al., 1998).

\section{A "sinergia de pragas" ou "AIDS como sindemia"}

Como sugerido pelo trabalho de Castells (1998) a respeito da globalização do capitalismo informacional, uma das tendências-chave nos anos recentes em termos sóci o-econômicos foi a polarização da desigualdade não apenas nos 
chamados "países em desenvolvimento" mas também naqueles denominados "desenvolvidos". De acordo com esta tendência, à medida que a reestruturação econômica foi-se desenrolando em países como os Estados Unidos, produziu-se significativa redução nas oportunidades de emprego para as populações de baixa renda e menor instrução formal - como os grupos de minorias que se concentraram nas áreas mais internas, o chamado inner city, dos centros urbanos mais importantes (Wilson, 1996).

Nesta América das áreas internas das grandes cidades, tanto quanto na África subdesenvolvida, o resultado foi a concentração da pobreza em certas comunidades que se tornaram, em grande medida, irrelevantes para o processo de desenvolvimento do capitalismo - e, na esteira de níveis crescentes de pobreza e miséria, explodiu um conjunto de problemas associados, ligados ao bem-estar social. As extensas investigações sociais e antropológicas que têm sido conduzidas nas comunidades das áreas internas das grandes cidades dos Estados Unidos - assim como os trabalhos relativos à pobreza, migração e desigual dade de gênero em uma gama de países em desenvolvimento são, em princípio, diretamente relevantes para a análise das ligações entre pobreza, desigualdade e HIV/ AIDS no Brasil.

A partir do fim dos anos 80, por exemplo, autores como Rodrick Wallace, Deborah Wallace, Mindy Fullilove, Robert Fullilove e Peter Gould exploraram a interação de uma série de problemas sociais que afetavam as vizinhanças habitadas por membros de minorias das áreas internas das grandes cidades dos Estados Unidos. Eles focalizaram, em particular, o impacto da redução de um conjunto de serviços essenciais - como serviços de combate a incêndio para comunidades de baixa renda - e documentaram os modos pelos quais as alterações no fornecimento destes serviços levaram a níveis crescentes de "desertificação urbana" em tais comunidades.

A desertificação urbana - caracterizada, por exemplo, por habitações incendiadas ou demolidas para dar passagem à construção de vias expressas - levou ao abandono destas vizinhanças por parte de qualquer um com recursos para fazê-lo, deixando para trás apenas os residentes mais pobres e destituídos, criando efetivamente uma "terra de ninguém", na qual os aspectos regulares da ordem social - como o policiamento efetivo - funcionam precariamente ou não existem, e onde níveis extremamente elevados de mortalidade por violência, tráfico de drogas e abuso de substâncias ilegais, mortalidade infantil e infecção pelo HIV têm sido documentados (Wallace, 1988, 1990, 1991a, 1991b; Wallace et al., 1994; Wallace \& Wallace, 1995).

Com base em tais análises, Wallace et al. (1994, 1995) têm argumentado que tais comunidades empobrecidas das áreas internas das grandes cidades são cada vez mais caracterizadas por "sinergia de pragas", na qual os efeitos negativos de uma gama de problemas sociais diferentes - relacionados ao déficit habitacional e de serviços ligados ao bem-estar social tanto quanto à carência ou inadequação de serviços de saúde - interagem sinergicamente para criar vulnerabilidade aumentada ao HIV e à AIDS (Wallace, 1988).

Tais análises têm si do reforçadas e estendidas em, pelo menos, duas direções importantes. Em primeiro lugar, algumas pesquisas têm enfocado a importância da raça ou etnicidade em tais comunidades das áreas internas das grandes cidades, examinando os efeitos, no tocante à saúde, da discriminação étnica e racial e enfatizando as inter-relações entre alcoolismo, abuso de drogas e infecção pelo HIV entre populações afro-americanas e latino-americanas marginalizadas e social mente excluídas. De modo semelhante a Wallace, Merrill Singer, em seu trabal ho a respeito da crise na saúde enfrentada pela população pobre de área urbana nos Estados Unidos, tem falado do HIV/ AIDS como uma espécie de "sindemia", que interage negativamente com os demais problemas de saúde enfrentados por esta população, produzindo níveis ainda maiores de vulnerabilidade (Singer, 1994; Baer et al., 1997).

Paul Farmer, Margaret Connors e Janet Simmons associaram tais problemas à questão do gênero, examinando os efeitos interativos daquilo que é descrito como "violência estrutural", que vincula pobreza e gênero - tanto em contextos desenvolvidos/industrializados quanto nos subdesenvolvidos/ em industrialização situando as mulheres mais pobres no que talvez sejam as condições mais extremas de vulnerabilidade enfrentadas por qualquer grupo populacional (Farmer, 1999; Farmer et al., 1996).

No contexto mais geral de decaimento e empobrecimento urbano encontrado nos Estados Unidos - em particular, nas cidades ao longo da costa Leste daquele país -, os analistas têm procurado explicitar os modos pelos quais a interação entre pobreza, racismo, opressão de gênero e todo um cortejo de males sociais se assemelha às relações encontradas em pesquisas desenvolvidas nos países mais pobres (Singer, 1998).

Muito da discussão acerca das transformações que vêm ocorrendo nas áreas internas das 
grandes cidades dos Estados Unidos tem enfatizado o que al guns analistas designam como "terceiromundização" destas comunidades. De fato, não sem algum grau de hipérbole, mas nem por isso de forma menos intrigante, em alguns veículos de imprensa surgi u a expressão "brasilianização", referindo-se às alterações nas relações sociais e de trabalho nos Estados Unidos, sugerindo um conjunto importante de conexões entre os fenômenos que foram examinados com relação ao HIV/ AIDS nas populações empobreci das das áreas internas das grandes cidades americanas e as tendências observadas no que concerne às mudanças do contorno da epidemia de HIV/ AIDS no Brasil.

Na seção final deste texto, tentaremos delinear algumas das implicações do conjunto de publicações produzido por estas pesquisas no tocante ao entendimento mais abrangente da epidemia de HIV/AIDS em sua evolução no Brasil e quanto à possibilidade de responder a esta evolução de forma mais eficiente no futuro.

\section{Delineando uma agenda de pesquisa e ação para o Brasil}

Talvez o ponto crucial a ser enfatizado com base nesta revisão, seja a existência de um conjunto expressivo de publicações nas áreas de antropologia e sociologia que pode apoiar a construção de base teórica e conceitual para o estudo empírico das transformações do contorno da epidemia de HIV/ AIDS no Brasil, bem como das tendências emergentes associadas à pauperização, feminização e interiorização evidenciadas a partir dos dados epidemiológicos.

Em particular, chamamos a atenção para a importância dos trabal hos recentes a respeito de: a) processo de globalização e reestruturação social e econômica do sistema capitalista mundial; b) literatura baseada em pesquisas transculturais relativa aos fatores ambientais e estruturais que conformam a epidemia (ou epidemias) de HIV/AIDS no contexto de vários países em desenvolvimento, e c) investigação detalhada das dimensões geográficas, culturais e sociais do HIV/AIDS nas populações empobrecidas e marginalizadas encontradas nos centros urbanos mais importantes da América do Norte. Tais linhas de trabal ho constituem três importantes conjuntos de pesquisas que oferecem insights diretamente rel evantes para a análise, investigação e intervenção com relação ao caráter mutante da epidemia no Brasil.

Com efeito, o Brasil pode ser visto como complexa síntese em que estão virtualmente presentes todos os diferentes fatores socioeco- nômicos identificados como estruturantes da vulnerabilidade relacionada à AIDS. Tanto pelo grau de heterogenei dade como de desigual dade, o Brasil repete, em escala nacional, os processos que autores como Castells descrevem no âmbito global. Formas variadas de desigualdade e opressão, conjuntamente com variada gama de fatores estruturais e ambientais, combinam-se no território nacional do Brasil para produzir e reproduzir forças sociais que foram identificadas, em âmbito internacional, como motores da epidemia: os movimentos migratórios das mulheres de Gana, citados acima, por exemplo, podem ocorrer internamente, no País, entre o nordeste e o sul-sudeste; a falta de poder das mulheres sujeitas simultaneamente à opressão econômica e à opressão de genêro repete-se tanto nas grandes cidades quanto nas áreas rurais do país; o encolhimento do mercado formal de trabalho e a desintegração social produzi da pelo crime organizado e o tráfico de drogas fazem parte da vida contemporânea brasileira da mesma forma que da vida novaiorquina; e assim por diante.

Ao vasto conjunto de desigual dades sociais e econômicas encontrado hoje no Brasil, devese agregar fatores igualmente agravantes, tais como a baixa incorporação dos valores da cidadania, menor ainda para os despossuídos, que acaba constituindo complicador adicional no caso brasileiro - o nosso gueto não conta com uma NAACP - National Association for the Advancement of Colored People, entidade norte-americana pioneira na defesa das minorias de origem africana naquele país - ou entidades semel hantes que lutam, com grande expressão social, pelos direitos sociais e políticos dos excluídos. A sinergia aqui encontrada inclui, ao contrário, a falta quase absoluta de mecanismos de proteção social e um sistema de bemestar social abortado quase antes de existir.

Em suma, enquanto virtualmente todos os fatores estruturais e ambientais associados à vulnerabilidade aumentada ao HIV/AIDS - em particular, entre os pobres - estão presentes no Brasil de hoje - muitas vezes em expansão -, dentre as estruturas potencial mente mitigadoras do impacto destes fatores, como os sistemas de bem-estar e as redes de proteção social - existentes em algumas das sociedades descritas nos estudos revisados -, poucas parecem de fato em operação no país, de modo que pudesse efetivamente auxiliar na resposta às fontes potenciais de vulnerabilidade.

Precisamente por causa disto, a bibliografia aqui revisada concernente às dimensões sociais e antropológicas da pobreza com relação ao HIV/ AIDS oferece certo número de insights 
importantes com relevância direta para que se possa estabelecer recomendações sobre o desenvolvimento futuro de pesquisas, intervenções, políticas e programas relacionados à AIDS no Brasil.

Uma discussão exaustiva destas implicações requereria um texto bem mais extenso do que é possível aqui. Vale a pena, no entanto, destacar pelo menos três pontos centrais, juntamente com al guns desdobramentos a eles associados.

Em primeiro lugar, gostaríamos de enfatizar a necessidade urgente de desenvolver quadros conceituais de referência mais sofisticados para a discussão da desigualdade com relação ao HIV/AIDS. A necessidade de maior sofisticação conceitual não é, de forma al guma, novidade. Está evidente, por exemplo, na importante redefinição das noções de risco individual ou grupal em termos de sistemas de vulnerabilidade social, noção esta que serviu de ponto de partida para este ensaio e que tem sido objeto de discussão teórica extensa e importante (Mann et al., 1993; Ayres, 1994; Mann \& Tarantola, 1996; Parker, 1996; Ayres et al., 1999). Isto é especialmente verdadeiro no que diz respeito à relação entre AIDS e pobreza, expressão de uso cada vez mais ampliado - quase um bordão -, mas com elaboração conceitual relativamente restrita.

A discussão destes tópicos carece de delineamento teórico-conceitual direto e preciso como aquele oferecido por autores como Castells na sua discussão a respeito da diferenciação social, discutida anteriormente (Castells, 1998). Um refinamento das ferramentas teórico-conceituais - que permita, por exemplo, estabelecer relações consistentes entre desigualdade geral e pobreza, desigual dade e polarização, pobreza e miséria, exploração extrema e exclusão social ou ainda exclusão social e integração perversa - ajudaria a ultrapassar as generalizações superficiais que freqüentemente caracterizam as discussões relativas à AIDS eà pobreza, de modo a desenvolver referenciais analíticos mais complexos e potencialmente mais úteis, com os quais se possa conduzir tanto a investigação quanto a intervenção no futuro.

Lembrando Bachelard (filósofo francês 1884-1963), o estado atual da discussão, pela proliferação de banalidades, constitui, de fato, um obstáculo epistemológico a este avanço.

Uma segunda lição importante a ser enfatizada, com base na bibliografia aqui examinada, diz respeito a em que medida a pobreza, por si mesma, é ao menos parcialmente limitada como categoria unicausal de análise da vulnerabilidade ao HIV/AIDS. Ao contrário, virtualmente toda a bibliografia sociológica e an- tropológica por nós revisada sugere que a pobreza (mesmo quando re-significada com mais complexidade como miséria, exclusão social, integração perversa e assim por diante) funciona, em quase todas as circunstâncias, em conjunção com um leque de outros fatores sociais e culturais na articulação de formas variadas de vulnerabilidade ao HIV/AIDS.

Em toda esta bibliografia, a ênfase constante é na interação entre fatores estruturais diversos - a sinergia causada pelo contato entre os fatores. Esta bibliografia aborda a pobreza em relação ao deslocamento espacial, ao poder associado a gênero, à violência e discriminação sexual, à desertificação urbana e à desintegração social associada ao tráfico de drogas e assim por diante. Tendências amplas, como a pauperização, a feminização ou a interiorização são inegáveis, mas nossa utilização, por vezes, demasiado simplista destas categorias pode mascarar a real complexidade social dos processos de vulnerabilização.

A discussão relativa à feminização, por exemplo, usualmente mascara não apenas o fato de que nem todas as mulheres são vulneráveis; deixa-se também de lado que a vulnerabilidade diferencial afeta mulheres diferentes de modo diverso, dependendo de outros fatores estruturais, ou seja, que mulheres pobres são mais vulneráveis que as que não o são, que as mulheres pobres jovens são mais vulneráveis que as mais velhas pertencentes ao mesmo estrato, que as mulheres pobres jovens sem alternativas econômicas viáveis além do trabalho com sexo são mais vulneráveis do que aquelas que dispõem de outras opções econômicas e assim por diante.

Da mesma forma, a ênfase na pauperização e feminização é lida, com freqüência, não apenas no noticiário da mídia, mas mesmo em círculos da saúde pública como sugerindo que o HIV/AIDS foi de algum modo resolvido entre os homens que fazem sexo com homens, mascarando o fato concreto de que a epidemia neste segmento populacional, da mesma forma que em outros, continua em seu curso, tendo sido simplesmente rearticulada, de modo que afeta, de preferência, os mais empobrecidos dentre os homens que fazem sexo com homens ou, neste grupo, aqueles que estão excluídos do mercado de trabal ho, ao invés de todos os homens que fazem sexo com homens. Enquanto homens brancos de classe média que se identificam como gays talvez estejam, de fato, deparando-se com situação de vulnerabilidade à AIDS menor que no passado, o mesmo provavelmente não é verdade para o travesti jovem, negro, da periferia suburbana. 
Ao fim e ao cabo, tais relações sinérgicas - e a complexidade incômoda que elas necessariamente causam aos esquemas bem arrumados da saúde pública, da epidemiologia e da ciência em geral - devem necessariamente ser confrontadas se pretendemos ir adiante na análise da epidemia ou na intervenção em sua trajetória.

Finalmente, avançar na utilização de quadros referenciais mais complexos, visando ao entendimento mais amplo das relações sinérgicas entre a pobreza e outras formas de opressão, desigualdade e exclusão social, demandará, por seu turno, al guns deslocamentos fundamentais em um conjunto razoavelmente entranhado de práticas que evoluíram ao longo do tempo e que, deste modo, se tornaram crescentemente assumidas como dadas e não questionadas na programação, pesquisa e intervenção vinculadas à AIDS.

Em alguns casos, estas mudanças deveriam ser de efetivação relativamente simples, como, por exemplo, a necessidade de iniciar a coleta de dados e trabalhar os bancos de dados de modo a incluir, de fato, indicadores sociais, demográficos, étnicos, econômicos etc. para que possamos dispor de parâmetros que possibilitem analisar questões sociais e econômicas como as que foram apontadas aqui. Em outros casos, tais mudanças podem ser mais difíceis, uma vez que vão de encontro não apenas à prática estabelecida, mas também a relações profundamente enraizadas de poder, dominação e mesmo, por vezes, de dependência, tal como se desenvolveram nas práticas científicas e na construção de programas de controle e prevenção da AIDS, como a necessidade de maior criatividade no que diz respeito às pesquisas financiadas pelo poder público (e propostas pela comunidade científica brasileira) com recursos nacionais tanto quanto internacionais, rompendo com os anéis de ferro do behaviorismo.

Principalmente em virtude de os modelos behavioristas dominarem a agenda de pesquisa em quase todas as agências internacionais, e nos principais centros de pesquisa sobre AIDS nos Estados Unidos e muitos países na Europa, modelos mais sofisticados e capazes de incorporar a análise dos fatores aqui levantados caminham na contramão não somente da hegemonia econômica da ordem econômica e política estabelecida, mas também da hegemonia epistemológica da ordem científica. Promover transformação neste sentido enfrentará fortes resistências de diversas instâncias.

Finalmente, o reconhecimento de tais fatores em sua plenitude demandará o repensar não apenas dos modos como conduzimos a pesquisa que busca entender a epidemia de HIV/ AIDS, mas também dos meios que utilizamos para intervir em resposta a ela. Um passo absolutamente essencial neste rearranjo será o estímulo à pesquisa e intervenção com maior base comunitária como a chave para a implementação de programas. Até aqui, muito da intervenção - e, provavelmente, mais ainda da pesquisa - tem enfocado os grupos abrangentes, com base populacional, mais do que a mobilização e transformação fundada em comunidades. Em alguns casos, como entre os homens que fazem sexo com homens, tal trabalho se mostra associado à formação de comunidades novas e importantes (Parker, 1999) e, em conseqüência, teve resultados talvez inesperados e, em geral, muito positivos. Ainda assim, muitos destes trabal hos, mesmo quando implementados por ONGs, falharam em enfocar as reais necessidades das comunidades afetadas - em especial, as comunidades empobrecidas e marginalizadas - de modo expressivo. A implementação de atividades ligadas a estes projetos, tanto através de ONGs quanto de agências governamentais, comumente não conta com base comunitária significativa.

Mais ainda, embora a relação entre o setor governamental e as ONGs/ AIDS tenha experimentado progressiva melhora ao longo dos anos, ainda persiste um modelo de interação moldado à imagem de outras interações institucionais, como, por exemplo, o financiamento de pesquisas por parte do Consel ho Nacional de Desenvolvimento Científico e Tecnológico - CNPq ou o próprio financiamento da assistência no SUS, em que o Estado assume o papel de comprador de serviços e auditor da prestação de contas sem integração efetiva de ações. Neste sentido, modelos como os programas de ações básicas de saúde ou de saúde da família podem oferecer exemplos interessantes de práticas inovadoras na relação Estado/ sociedade civil. Infelizmente, contudo, ainda é baixa a integração destes programas com as demais ações do poder público na área da saúde, aí incluindo-se o Programa de AIDS e o próprio SUS. Precisamente por conta da complexidade da "indústria da AIDS" (Patton, 1991) tal como esta evoluiu, ao longo do tempo, no Brasil e em outras partes do mundo - desenvolver novas modalidades de abordagem para estes problemas não será, de modo algum, tarefa simples e seguramente despertará resistências apreciáveis por vários motivos.

Para que a intervenção seja de fato efetiva em meio às circunstâncias que analisamos aqui, deve basear-se em concepção mais completa das múltiplas dimensões que precisam 
ser agregadas. Por exemplo, é necessário garantir a integração da assistência à saúde às pessoas com HIV/ AIDS com medidas de proteção social (jurídica, assistência social etc.); é preciso desenvolver intervenções focalizadas nas situações de maior vulnerabilidade (como trabalho infantil, inclusive a prostituição) e suporte social para excluídos, muitas vezes por fatores múltiplos (por exemplo, os travestis pobres ou outros grupos marginalizados por di-

\section{Agradecimentos}

Este trabalho foi financiado pela Coordenação Nacional de DST e AIDS do Ministério da Saúde.

Referências

ABIA (Associação Brasileira Interdisciplinar de AIDS), 1999. Métodos controlados pelas mulheres. Boletim ABI A Especial, 43.

AGGLETON, P., 1996. Global priorities for HIV/ AIDS intervention research. International Journal of Sexually Transmitted Diseases and AIDS, 7 (Sup. 2):13-16.

AKEROYD, A. V., 1994. HIV/ AIDS in Eastern and Southern Africa. Review of African Political Economy, 60:173-184.

ANARFI, J., 1993. Sexuality, migration and AIDS in Ghana: A socio-behavioral study. Health Transition Review, 3 (Sup.):45-67.

ARCHAVANITKUL, K. \& GUEST, P., 1994. Migration and the commercial sex sector in Thailand. Health Transition Review, 4 (Sup.):273-295.

AYRES, J. R. C. M., 1994. Epidemiologia sem números: Outras reflexões sobre a ciência epidemiológica, a propósito da AIDS. In: Seminário Epidemiologia Social da AIDS, Anais, pp. 8-19, Rio de Janeiro: ABIA/IMS-UERJ.

AYRES, J. R. C. M., 1996. O jovem que buscamos e o encontro que queremos ter: A vulnerabilidade como eixo de avaliação de ações preventivas de abuso de drogas, DST e AIDS entre crianças e adolescentes. In: Papel de Educação na Ação Preventiva ao Abuso de Drogas eàs DST/AIDS (D. A. Tozzi, N. L. Santos, C. M. Amarao, E. Almeida, E. J. Silva \& M. L. Pereira, eds.), pp. 15-24, São Paulo: Fundação para o Desenvolvimento da Educação.

AYRES, J. R. C. M.; FRANÇA Jr., I.; CALAZANS, G. J. \& SALETTI FILHO, H. C., 1999. Vulnerabilidade e prevenção em tempos de AIDS. In: Sexualidades pel o Avesso: Direitos, Identidades e Poder (R. M. Barbosa \& R. Parker, eds.), pp. 49-72, São Paulo: Editora 34/Rio de Janeiro: Instituto de Medicina Social, Universidade do Estado do Rio de Janeiro.

BAER, H.; SINGER, M. \& SUSSER, I., 1997. Medical Anthropology and the World System. Westport: Bergin \& Garvey. versos fatores e por meio de diversos eixos de opressão). No fim das contas, a intervenção em resposta ao HIV/ AIDS dependerá não apenas das ações técnicas da saúde pública, mas de nossa capacidade mais ampla em contribuir para transformações sociais verdadeiramente progressistas - em um mundo no qual as transformações mais amplas que se desenrolam ao nosso redor parecem freqüentemente estar caminhando na direção oposta.

BARBOSA, R. M., 1999. Negociação sexual ou sexo negociado? Poder, gênero e sexualidade em tempos de AIDS. In: Sexualidades pelo Avesso: Direitos, Identidades e Poder (R. M. Barbosa \& R. Parker, eds.), pp. 73-88, São Paulo: Editora 34/Rio de Janeiro: Instituto de Medicina Social, Universidade do Estado do Rio de Janeiro.

BASSETT, M., 1993. Women in Zimbabwe: A Case Study. WHO/GPA Meeting on Enabling Approaches in HIV/ AIDS Prevention. Geneva: World Health Organization. (mimeo.)

BASTOS, F. I., 1996. Ruína \& Reconstrução: AIDS eDrogas Injetáveis na Cena Contemporânea. Rio de Janeiro: Editora Relume-Dumará/ ABIA/IMS-UERJ.

BOND, G. C. \& VINCENT, J., 1991. Living on the edge: Structural adjustment in the context of AIDS. In: Changing Uganda: The Dilemmas of Structural Adjustment and Revolutionary Change(H. B. Hansen $\&$ M. Twaddle, eds.), London: James Curry.

CARAËL, M.; BUVÉ, A. \& AWUSABO-ASARE, K., 1997. The making of HIV epidemics: What are the driving forces? AIDS, 11 (Sup. B):S23-S31.

CASTILHO, E. \& CHEQUER, P., 1997. Epidemiologia do HIV/ AIDS no Brasil. In: Políticas, InstituiçõeseAIDS: Enfrentando a Epidemia no Brasil (R. Parker, ed.), pp. 17-42, Rio de Janeiro: Jorge Zahar Editor/ABIA.

CASTELLS, M., 1999. A Era da Informação. I: A Sociedadeem Rede. Rio de Janeiro: Paz eTerra.

CASTELLS, M., 1998. The Information Age, III: End of Millenium. Oxford: Blackwell.

CNDST/AIDS (Coordenação Nacional de Doenças Sexualmente Transmissíveis e AIDS), 1999. Boletim Epidemiológico - AIDS, Semana Epidemiológica de 09 a 21. XII (02).

DANIEL, H. \& PARKER, R., 1991. AIDS: A Terceira Epidemia. São Paulo: Iglu.

DANIEL, H. \& PARKER, R., 1993. Sexuality, Politics and AIDS in Brazil. London: The Falmer Press.

DECOSAS, J., 1996. HIV and development. AIDS, 10 (Sup. 3):S69-S74. 
DECOSAS J.; KANE, F.; ANARFI, J. K.; SODJI, K. D. \& WAGNER, H. U., 1995. Migration and AIDS. Lancet, 346:826-828.

DES JARLAIS, D. C.; FRIEDMAN, S.; CHOOPANYA, K.; VANICHSENI, S. \&WARD, T. P., 1992. International epidemiology of HIV and AIDS among injecting drug users. AIDS, 6:1.053-1.068.

ELIAS, C. \& HEISE, L., 1994. Challenges for the development of the female-controlled vaginal microbicides. AIDS, 8:1-9.

FARMER, P., 1992. AIDS and Accusation: Haiti and the Geography of Blame. Berkeley: University of California Press.

FARM ER, P., 1995. Culture, poverty and the dynamics of HIV transmission in rural Haiti. In: Cultureand Sexual Risk: Anthropological Perspectives on AIDS (H. Brummelhuis \& G. Herdt, eds.), pp. 3-28, New York: Gordon \& Breach.

FARMER, P., 1999. Infections and Inequalities. Berkeley: University of California Press.

FARMER, P.; LINDENBAUM, S. \& GOOD, M. D., 1993. Women, poverty and AIDS: An introduction. Culture, Medicine, and Psychiatry, 17:387-397.

FARMER, P.; CONNORS, M. \& SIMMONS, J., 1996. Women, Poverty, and AIDS: Sex, Drugs and Structural Violence. Monroe: Common Courage Press.

GOMES, M. R. O., 1998. Epidemia da AIDS no Brasil: Atualização. Boletim Epidemiológico - AIDS, XI (4).

GUPTA, G. R. \& WEISS, E., 1993. Women's lives and sex: Implications for AIDS prevention. Culture, Medicine, and Psychiatry, 17:399-412.

HARVEY, D., 1989. The Condition of Postmodernity. Oxford: Blackwell.

HEISE, L. \& ELIAS, C., 1995. Transforming AIDS prevention to meet women's needs: A focus on developing countries. Social Scienceand Medicine, 40: 931-943.

JOCHELSON, K.; M OTHIBELI, M. \& LEGER, J. P., 1991. HIV and migrant labour in South Africa. International Journal of Health Services, 21:157-170.

FARM ER, P., 1995. Culture, poverty and the dynamics of HIV transmission in rural Haiti. In: Cultureand Sexual Risk: Anthropological Perspectives on AIDS (H. Brummelhuis \& G. Herdt, eds.), pp. 3-28, New York: Gordon \& Breach.

KRENISKE, J., 1997. AIDS in the Dominican Republic: Anthropological reflections on the social nature of disease. In: AIDS in Africa and the Caribbean (G. C. Bond, J. Kreniske, I. Susser \& J. Vincent, eds.), pp. 33-50, Boulder: Westview Press.

LARVIE, P., 1997. Homophobia and the ethnoscape of sex work in Rio de Janeiro. In: Sexual Cultureand Migration in the Era of AIDS: Anthropological and Demographic Perspectives (G. Herdt, ed.), pp. 143164, Oxford: Clarendon.

LINDENBAUM, S., 1998. Images of catastrophe: The making of an epidemic. In: The Political Economy of AIDS (M. Singer, ed.), pp. 33-58, Amityville: Baywood Publishing Co.

LURIE, P.; HINTZEN, P. \& LOWE, R. A., 1995. Socioeconomic obstacles to HIV prevention and treatment in developing countries: The roles of the International Monetary Fund and the World Bank. AIDS, 9:539-546.

MANN, J. M.; TARANTOLA, D. \& NETTER, T. W., 1993. A AIDS no Mundo. Rio de Janeiro: Editora Relume-Dumará.
MANN, J. M. \& TARANTOLA, D., 1996. AIDS in the World II. New York/Oxford: Oxford University Press.

MCKENNA, N., 1996. On the Margins: Men who Have Sex with Men and HIV in Developing Countries. Londres: Panos Institute.

MICHAL-JOHNSON, P., 1994. The dark side: Barriers to changing high-risk behaviors. AIDS and Public Policy, 9:18-19.

PAIVA, V., 1996. Fazendo Arte com Camisinha: A História de um Projeto de Prevenção de AIDS para Jovens. Tese de Doutorado, São Paulo: Faculdade de Psicologia, Universidade de São Paulo. (mimeo.)

PAIVA, V., 1999. Cenas sexuais, roteiros de gênero e sujeito sexual. In: Sexualidades pelo Avesso: Dire itos, Identidades e Poder (R. M. Barbosa \& R. Parker, eds.), pp. 247-269, São Paulo: Editora 34/Rio de Janeiro: Instituto de Medicina Social, Universidade do Estado do Rio de Janeiro.

PARKER, R., 1993. The negotiation of difference: Male prostitution, bisexual behavior and HIV transmission in Brazil. In: Sexuality, Politics and AIDS in Brazil (H. Daniel \& R. Parker, eds.), pp. 85-96, London: The Falmer Press.

PARKER, R., 1996. Empowerment, community mobilization and social change in the face of HIV/ AIDS. AIDS, 10 (Sup. 3):S27-S31.

PARKER, R., 1997. Migration, sexual subcultures, and HIV/AIDS in Brazil. In: Sexual Culture and Migration in the Era of AIDS: Anthropological and Demographic Perspectives (G. Herdt, ed.), pp. 55-69, Oxford: Clarendon.

PARKER, R., 1999. Beneath the Equator: Cultures of Desire, Male Homosexuality, and Emerging Gay Communities in Brazil. Nova York: Routledge.

PARKER, R. \& GALVÃO, J., 1995. Quebrando o Silêncio: Mulheres e AIDS no Brasil. Rio de Janeiro: ABIA/IMS-UERJ/Editora Relume-Dumará.

PARKER, R.; KHAN, S. \& AGGLETON, P., 1998. Conspicuous by their absence? Men who have sex with men (MSM) in developing countries: Implications for HIV prevention. Critical Public Health, 8:329-346.

PATTON, C., 1991. Inventing AIDS. New York: Routledge.

ROMERO-DAZA, N. \& HIMMELGREEN, D., 1998. More then money for your labor: Migration and the political economy of AIDS in Lesotho. In: The Political Economy of AIDS (M. Singer, ed.), pp. 185-204, Amityville: Baywood Publishing Co.

SCHOEPF, B., 1988. Women, AIDS, and the economic crisis in Central Africa. Canadian Journal of African Studies, 22:625-644.

SCHOEPF, B. G., 1992a. Gender relations and development: Political economy and culture. In: Twenty-First Century Africa: Toward a New Vision of Self-Sustainable Devel opment (A. Seidman \& F. Anang, eds.), Trenton: Africa World Press.

SCHOEPF, B. G., 1992b. Women at risk: Case studies from Zaire. In: The Time of AIDS: Social Analysis, Theory and Method (G. Herdt \& S. Lindenbaum, eds.), pp. 259-286, Beverly Hills: Sage Publications.

SCHOEPF, B. G.; WALU, E.; RUKANGIRA, W. N.; PAYANZO, N. \& SCHOEPF, C., 1991. Gender, power, and risk of AIDS in Central Africa. In: Women 
and Health in Africa (M. Turshen, ed.), pp. 187203, Trenton: Africa World Press.

SENNETT, R., 1999. A Corrosão do Caráter. Rio de Janeiro: Record.

SINGER, M., 1994. AIDS and the health crisis of the U.S. urban poor: The perspective of critical medical anthropology. Social Science and Medicine, 39:931-948.

SINGER, M., 1998. The Political Economy of AIDS. Amityville: Baywood Publishing Co.

STEIN, Z. A., 1990. HIV prevention: The need for methods women can use. American Journal of Public Health, 80:460-462.

SUSSER, I. \& KRENISKE, J., 1997. Community organizing around HIV prevention in rural Puerto Rico. In: AIDS in Africa and the Caribbean (G. C. Bond, J. Kreniske, I. Susser \& J. Vincent, eds.), pp. 51-64, Boulder: Westview Press.

SWEAT, M. D. \& DENNISON, J. A., 1995. Reducing HIV incidence in developing countries with structural and environmental interventions. AIDS, 9 (Sup. A): S251-S257.

SYM ONDS, P. V., 1998. Political economy and cultural logics of HIV/AIDS among the Hmong in Northern Thailand. In: The Political Economy of AIDS (M. Singer, ed.), pp. 205-266, Amityville: Baywood Publishing Co.

TAN, M. L., 1993. Socioeconomic impact of HIV/ AIDS in the Philippines. AIDS Care, 5:283-288.

TAWIL, O.; VERSTER, A. \& O'REILLY, K. R., 1995. Enabling approaches for HIV/ AIDS prevention: Can we modify the environment and minimize the risk? AIDS, 9:1299-1306.

TURSHEN, M., 1995. Response: Societal instability in international perspective: Relevance to HIV/AIDS Prevention. In: Assessing the Social and Behavioral Science Base for HIV/AIDS Prevention and Intervention [Workshop Summary], pp. 117-128, Washington, D.C.: National Academy Press.

TURSHEN, M., 1998. The political ecology of AIDS in Africa. In: The Political Economy of AIDS (M . Singer, ed.), pp. 169-184, Amityville: Baywood Publishing Co.
WALLACE, R., 1988. A synergism of plagues: “Planned shrinkage", contagious housing destruction and AIDS in the Bronx. Environmental Research, 47:133.

WALLACE, R., 1990. Urban desertification, public health and public order: "Planned shrinkage," violent death, substance abuse and AIDS in the Bronx. Social Science and Medicine, 31:801-813.

WALLACE, R., 1991a. Traveling waves of HIV Infection on a low dimensional "socio-geographic" network. Social Scienceand Medicine, 32:847-852.

WALLACE, R., 1991b. Social disintegration and the spread of AIDS: Thresholds for propagation along "socio-geographic" networks. Social Science and Medicine, 33:1155-1162.

WALLACE, R.; FULLILOVE, M. T.; FULLILOVE, R.; GOULD, P. \& WALLACE, D., 1994. Will AIDS be contained within U.S. minority urban populations? Social Science and Medicine, 39:1051-1062.

WALLACE, R. \& WALLACE, D., 1995. U.S. apartheid and the spread of AIDS to the suburbs: A multicity analysis of the political economy of spatial epidemic threshold. Social Science and Medicine, 41:333-345

WATTERS, M., 1995. Globalization. London: Routledge.

WEBB, D., 1997. HIV and AIDS in Africa. London: Pluto Press.

WILSON, W. J., 1996. When Work Disappears: The World of the New Urban Poor. New York: Vintage Books.

WILSON, D.; SIBANDA, B.; MBOYI, L.; MSIMANGA, S. \& DUBE, G., 1990. A Pilot Study for an HIV prevention program among commercial sex workers in Bulawayo, Zimbabwe. Social Science and Medicine, 31:609-618.

ZOYSA, I.; SWEAT, M . \& DENNISON, J., 1996. Faithful but fearful: Reducing HIV transmission in stable relationships. AIDS, 10 (Sup. A):S197-S203. 